\title{
Study on the Application of Integrated Simulation Technology in the First Aid Skills Training Zhaomin Qin ${ }^{1, a}$, Qinhai Shen ${ }^{1,{ }^{*}}$,Ruixue Kong ${ }^{1}$ \\ ${ }^{1}$ Shandong Medical College, Jinan, 250002, China \\ aqinqin@126.com \\ *Corresponding author shengqinghai@126.com
}

Keywords: Integrated simulation technology; Emergency care; Teaching

\begin{abstract}
Objective: To observe and analyze the effect of the application of the comprehensive simulation technology in emergency nursing teaching. Methods: We select our hospital nursing professional 200 nurses were divided into experimental group and control group. The experimental group carries out the high emulation integrated simulation to the first aid skills training on the basis of the traditional teaching, and the control group is according to the traditional teaching method. Results: The experimental group and control group in the assessment results were $89.5 \pm 3.6$ points, 83.6 \pm 4.7 points, the comparative difference was statistically significant $(\mathrm{P}<0.05)$, and $98 \%$ of nursing students think introducing simulation teaching method in emergency nursing experimental teaching is necessary. Conclusion: Through the first aid skills, an integrated simulation technology in teaching can help improve the comprehensive emergency ability of nursing students.
\end{abstract}

\section{Introduction}

The simulation teaching is to point to in under the guidance of teachers, nurses play a different role in a specific scenario simulation comprehensive skill training of a kind of teaching method. Simulation teaching to a certain extent, make up for the deficiencies of traditional emergency nursing experimental conditions, the approximate real training environment for nursing students, improve the practice ability of nursing students, ability to analyze and solve problems. And first aid of traditional experiment teaching means a single, lack of comprehensive and designing experiments, ignore the cultivation of the comprehensive ability of nursing students, is not conducive to the development and cultivation of nursing students comprehensive care consciousness, emergency organization ability and the team cooperation spirit. The emergency department is the largest concentration of the critical patients, patients, disease complex; nursing students rarely have the opportunity to really operate in patients, which will affect the teaching quality. The development of medical simulation education can effectively solve the problem; a study shows that simulation teaching can improve the ability of emergency health care workers. We for the clinical practice of nursing students, under the arrangement of "high" simulation situation of emergency nursing teaching, received good teaching effect, now report as follows.

\section{Materials and Methods}

We select 200 nursing students of the nursing specialty, 10 nursing students are the male, and 190 nursing students are the female. The nursing students' age is the 19 to 22 years old, and average 20.8 years. 100 nursing students are as experimental group, 100 nursing students are the control group. They are only 3 year, and are enrolled in the college entrance examination scores, and have no obvious difference.

\section{Methods}

The experimental group and control group were taught by the same teachers using the same materials, the total number of hours were 54 class hours. The group theory is for 24 hours, training 
lesson 30 hours; the control theory class 30 hours, 24 hours training class. Two groups of individual skills training project, same experimental compression theory of six hours and increase the comprehensive first aid skills training six hours.

1) The construction of the simulation of clinical situations. In order to better display of emergency patients in the process of teaching physical, psychological and social issues related to process and treatment and nursing, often encountered in clinical practice of emergency cases collected after editing and standardize cases, nursing students can be in a real emergency situation experience changes in physiological processes, experience life, "live" the essence of repetitive training of clinical practical work process, clinical experience, and shall not affect the safety of the patients. Such as editor for the standardization of cardiac arrest patients, organ phosphorus pesticide poisoning, myocardial infarction, and uncontrolled hemorrhagic shock, complete and defibrillation, breathing machine, monitor, bandage homeostasis more supplies, urethral catheterization bag, etc.

2) The determination of the teaching content. Choice to solve the problem of people's health as the main line, to train nursing students of nursing service consciousness, practical ability, interpersonal skills and humanistic care ability for the purpose, content to have comprehensive knowledge, ability and quality, and to have (and challenging, stimulating nursing students desire for knowledge and curiosity, cultivation of nursing students interest in learning. Such as selection of uncontrolled hemorrhagic shock for the teaching content, nursing students observe the illness quickly, notify the doctor and executed the doctor's advice, using intravenous indwelling needle rapidly develop venous pathway, urethral catheter, etc., cultivation of nursing students the basic knowledge of nursing, the integrated use of clinical nursing knowledge and nursing students desire for knowledge.

3) The teacher's configuration. Teaching the teacher's comprehensive quality directly affects the effect of classroom teaching, good teaching teachers is first aid (care), teaching experts, can according to the evolution of the disease especially in different students take different measures in different end is to make a corresponding guide, analysis, induction, therefore, on the teacher to double teachers is most appropriate. Sufficient collective lesson preparation before class, of course, essential, including some students, for simulation, prepare lesson plans, case teaching process, the scheme for evaluation.

4) The preparation before class and form study groups. Teachers in 2 to 3 weeks before the commencement of the cases of class will inform nursing students, learning tasks and requirements. Coordinated team, 5-6 escort raw elect a team leader in each group, nursing students prepare for class, advance design possible problems and find a solution. A collaborative team to comprehensively consider the sex of the nursing students, knowledge, language expression ability, is advantageous to the individual success, and should be conducive to the company success. As in cardiopulmonary resuscitation (CPR) large rescue, the boy was assigned to each group to lay a good foundation for the implementation of effective heart pressure. Team members separately before the class to consult materials, discussion may encounter difficulties, integration team members' knowledge and wisdom, in order to improve the class efficiency.

5) The classroom implementation and teaching evaluation. By nursing students as the main body, give full play to the teaching of teachers' leading role. Teaching the "brief theory teaching (or) component technology practice and integrated simulation exercises, video analysis summary" of the international fashion model. Assessment for the unit with the team, and finally selects the best study group. Speaker teachers from the whole nursing and humanistic care level comments on the performance of the nursing students, timely praise the advantages in the process of training, points out the problems and give guidance.

\section{Results}

The experimental group and control group in the assessment results were $89.5 \pm 3.6$ points, $83.6 \pm 4.7$ points, the comparative difference was statistically significant $(\mathrm{P}<0.05) .98 \%$ of nursing students think introducing simulation teaching method in emergency nursing experimental teaching is necessary. Compared two groups of nurses' theory result with the operating result. 
Table 1: Compared two groups of nurses theory result with the operating result

\begin{tabular}{ccc}
\hline Group & Number & Assessment Results \\
\hline The experimental group & 100 & $89.5 \pm 3.6$ \\
The control group & 100 & $83.6 \pm 4.7$ \\
$\chi 2$ & & 7.894 \\
P value & & $<0.05$ \\
\hline
\end{tabular}

\section{Discussion}

Comprehensive experiment is nursing students in the existing skills on the basis of preliminary experiment, improving the experiment skill and method of a compound experiment. Ever in emergency care experiments, emphasis on nursing students with cardiopulmonary resuscitation, homeostasis, bandage, fixation, handling emergency technology such as component, neglect of transfusion, sputum suction, oxygen, vital signs of test simulation of the real cases of a number of technical operations such as technology integration application, implement comprehensive experiments, nursing students group cooperate rescue training, individual training for a number of simulation training, increased the variety of condition of practice, nursing students has the feeling of immersive, shorten the distance teaching and clinical. The nursing is a practical subject and the domestic nursing education generally have more than the number of students, clinical practice teaching resources insufficiency, the less chance the student beginning. Results show that the ridge in the irrigation area of corridor width $40 \mathrm{~cm}, 15 \mathrm{~cm}$ deep groove, groove width of $15 \mathrm{~cm}$, grade 1:1 parameter combination for the optimal portfolio model of spring wheat), and the ridge $30 \mathrm{~cm}$ wide (T1) In recent years, high simulation teaching is widely used in the field of nursing education, but has yet to have a reliable and effective assessment tools, to confirm the real value of high simulation teaching degree and degree of effective and credible, and this will directly restricts the development of nursing education.

\section{The high-fidelity integrated simulation persons is applied to the background of the nursing teaching}

1) The growing tension of medical resources and students contradiction in today's domestic and foreign medical colleges and universities are faced with a dilemma: due to the lack of clinical nurses, health care proportion serious imbalance, the need for nurses to expanded to nursing students enroll in medical colleges, clinical practice are available for the base can not meet the demand of teaching effectively. As clinical practice nursing students more and qualified teaching nurses is relatively insufficient, this makes the nursing students of nursing operation is not effective in, can't guarantee each nursing students can get the same clinical learning experience.

2) The modern medical ethics and legal requirements with the development of the medical enterprise, the patient's ego to protect consciousness gradually strengthened, the patient has the right to say no to become the object of clinical trials. "People-oriented" the ethics also determines the unfavorable to the patient as a tool for improving nursing students' clinical skills test.

3) The hospital, the patient needs to have the high quality nursing talents with the development of science and technology changes with each passing day, more and more high-tech products and advanced medical instruments and equipment used in clinical, improvement of living standards allow the patient to clinical service quality requirements also increase, this requires before entering the clinical nurses who have the ability to provide high quality nursing service. Related studies show that nursing students in the process of to nurse is stressful and hard.

\section{The high-fidelity integrated simulation persons in nursing teaching application advantage}

1) To improve nursing students in clinical nursing skills high simulation laboratory can provide nursing students with a safe and controllable "clinical environment" and nursing students to simulate the implementation of various operations without having to worry about bring harm to the 
patient. As a result of the simulation system of repeatability, allow the nursing students to make mistakes, and through repeated practice in simulated man, until the master, in addition, for some patients with a rare and dangerous, nursing students in clinical difficult to come into contact with, can take advantage of the simulation system to create the opportunity to supply the practice nurses

In the absence of psychological pressure under the environment of study and master the relevant skills. After applying the high simulation in nursing experiments, the objective of clinical nursing students test (OSCF) performance is significantly higher than before the trial.

2) To improve nursing students clinical comprehensive quality of nursing students in high nursing experimental simulation to comprehensive utilization has been learned knowledge, play a critical thinking and creative thinking, the comprehensive evaluation on the "patient", to identify nursing problems of the "patient" and take appropriate nursing measures. "Patients" in the process of experimental condition can be changing, which requires a student nurse, observing the patients' condition and according to the "illness" change decisiveness, calm and orderly to provide care for the "patient", so as to effectively improve the nursing students of illness observation, clinical thinking ability and emergency response ability of clinical comprehensive ability, smooth implementation to the transformation of clinical nurses. Through the experimental results show that and did not receive high simulation were compared to the experimental group students to analyze and solve the problem of comprehensive ability has improved significantly.

3) It is helpful to improve the team spirit and communication ability of nursing students nursing students take part in the care of "patient" in the form of group, nursing students in the process of nursing through communication with "patient", "doctor" positive found in existing or potential nursing problems, team members a clear division of responsibilities, work together, common decision for "patient" to provide satisfactory nursing service. In the meeting, the discussion between teachers and students, the operation process of nursing students' communication and evaluation, to the deficiencies and the operator found itself to promote mutual learning between nursing students. Studies have found that most of the nursing students think participation of high simulation of nursing helps to cultivate team spirit, improve their ability of communication.

4) It is to improve nursing students learning initiative, enthusiasm and self-confidence. Since there is no standard nursing operation, nursing students need to use scientific nursing process and make use of the existent knowledge, arouse the enthusiasm of their own, actively, creatively provide nursing care for "patient", fully embodies the teaching idea of take the student as the main body. In addition, in conjunction with the technical staff, simulation people show some "physiological": such as cry of pain, answer the problems of nursing students, such as heart rate, recreating the clinical situation, greatly improved the nursing students' interest in learning. The experience of successful salvage "patient" can bring nursing students a sense of accomplishment and self-confidence, and prepare for the future clinical work. This survey is that $92 . \%$ of nursing students think high simulation nursing experiment to improve their self-confidence is a popular way of teaching.

\section{The high-fidelity integrated simulation people's application in the first aid skills teaching}

Through simulation people create true clinical scenarios, and simulate real emergency field, and comply with the stimulation of sound and picture and, for the protection of life to build a kind of nervous emergency atmosphere, can greatly improve the learning interest of nursing students. Cases by editors and Settings, in a limited amount of time without effective rescue, model people's illness will be developed to the direction of more dangerous and die, which requires the nursing students have to race against time to evaluate, judge and rescue, can well cultivate nursing students "life first, aging first" awareness of first aid. Team members throughout the rehearsal process to rational division of labor, close collaboration, for can't solve the problem, team members need to work together, mutual discussion, common to find the measures to solve the problem, in this way, nursing students in classroom activities to cultivate the good team cooperation spirit. These effects do more beyond the single operation training. 
Train the ability of nursing students in the humanities, has become the important content of nursing education in the 21st century. Have been reported, although humanistic care ability of nursing students in increase year by year, but still obviously insufficient, unit of choose and employ persons are not happy with the humanistic care ability of nursing students. Simulation teaching is by setting the humanistic care of the situation, make nursing students in close contact with the emergency patients, leading to the patient's sympathy and humanistic care consciousness, the cultivation of nursing students from the perspective of nursing, and realize humanistic care. Such as cultivation of nursing students in simulation teaching in nonverbal way, eye contact, facial expression and communication; In the patient's condition deteriorated, the cultivation of nursing students keep calm, don't too fast speed, lest cause the patient. At the end of each training teachers and students together to simulate the dirt from the face and body or stick in the body of the conductive adhesive dressed clean, clothes, quilt cover, consciously applying humanism spirit in practical work.

\section{Conclusion}

The simulation of high nursing experiment teaching has been as a kind of new teaching method, which is applied in modern nursing talents cultivation, and played a more and more important role. But at present there are still many problems to be solved: how to write a conform to the standardized nursing teaching characteristic simulation cases, how many time substitute simulation experiment for clinical practice is appropriate, how to objectively evaluate high simulation experiment can learn knowledge that eventually is translated into the real clinical behavior, etc., all these problems need to nursing educators continue to be explored and studied.

\section{References}

[1] Anderson LW, Krathwohl DR, Airasian PW, et al. A taxonomy for learning, teaching, and assessing: a revision of Bloom's taxonomy of educational objectives. 2011.

[2] Rumbaugh J, Blaha M, Premerlani W,et al. Object-Oriented Modeling and Design. 2012.

[3] Gant LT. Human Simulation in Emergency Nursing Education: Current Status. Journal of Emergency Nursing. 2012.

[4] Paula G H, Katherine SS, Christine AT,et al.A challenge to meet the future, Nursing education in Oregon. J Nuts Edu. 2012.

[5] Parr MB, Sweeney NM. Use of human patient simulation in an undergraduate critical care course. Critical Care Nursing Quarterly. 2012.

[6] Facione, P. A, Facion, N. C., Giancarlo, C. a. California critical thinking disposition inventory: Inventory Manual. 2011.

[7] Hall MJ, Adarno G, McCurry L, et al. Use of standardized patients to enhance a psychiatry clerkship. Academic Medicine . 2012.

[8] Susanne W Gibbons, Graceanne Adamo, Diane Padden, et al. Clinical evaluation in advanced Practice nursing education: Using standardized Patients in health assessment. Journal of Nursing. 2012.

[9] Mehnay WM, Lashley FR. Current use and opinions regarding human patientsimulators in nursing education: An international survey. Nursing Education Perspectives. 2011.

[10] McCausland LL, Curran CC, Cataldi P. Use of a human simulator forundergraduate nurse education. International Journal of Nursing Education Scholarship. 2012. 